\title{
Genomic Profiling by Whole-Genome Single Nucleotide Polymorphism Arrays in Wilms Tumor and Association with Relapse
}

\author{
Daniela Perotti, ${ }^{*}$ Filippo Spreafico, ${ }^{2}$ Federica Torri, ${ }^{3}$ Beatrice Gamba, ${ }_{7}$ Pio D'Adamo, ${ }^{4}$ Sara Pizzamiglio, ${ }^{5}$ \\ Monica Terenziani, ${ }^{2}$ Serena Catania, ${ }^{2}$ Paola Collini, ${ }^{6}$ Marilina Nantron, ${ }^{7}$ Andrea Pession, ${ }^{8}$ Maurizio Bianchi, ${ }^{9}$ \\ Paolo Indolfi, ${ }^{10}$ Paolo D'Angelo, ${ }^{11}$ Franca Fossati-Bellani, ${ }^{12}$ Paolo Verderio, ${ }^{5}$ Fabio Macciardi, ${ }^{3}$ and Paolo Radice ${ }^{1,13}$; \\ on behalf of the Associazione Italiana Ematologia Oncologia Pediatrica Wilms Tumor Working Group \\ 'Department of Preventive and Predictive Medicine, Unit of Molecular Bases of Genetic Risk and Genetic Testing, Fondazione IRCCS \\ Istituto Nazionale dei Tumori, Milano, Italy \\ ${ }^{2}$ Department of Medical Oncology, Pediatric Oncology Unit, Fondazione IRCCS Istituto Nazionale dei Tumori, Milano, Italy \\ ${ }^{3}$ Department of Psychiatry and Human Behavior, School of Medicine, University of California, Irvine, CA \\ ${ }^{4}$ Division of Medical Genetics, IRCCS Burlo Garofolo, University of Trieste, Trieste, Italy \\ ${ }^{5}$ Medical Statistics and Biometry Unit, Scientific Direction, Fondazione IRCCS Istituto Nazionale dei Tumori, Milano, Italy \\ ${ }^{6}$ Department of Pathology and Laboratory Medicine, Fondazione IRCCS Istituto Nazionale dei Tumori, Milano, Italy \\ ${ }^{7}$ Department of Pediatric Hematology and Oncology, Istituto G. Gaslini, Genova, Italy \\ ${ }^{8}$ Department of Pediatric Oncology and Hematology “Lalla Seràgnoli,' Clinica Pediatrica, Policlinico Sant'Orsola Malpighi, Bologna, Italy \\ ${ }^{9}$ Pediatric Department, Ospedale Infantile Regina Margherita, Torino, Italy \\ ${ }^{10}$ Pediatric Oncology, Pediatric Department, II University, Napoli, Italy \\ "Pediatric Hematology and Oncology Unit, A.R.N.A.S. Ospedali Civico, Di Cristina e Benfratelli, Palermo, Italy \\ ${ }^{12}$ Lega Italiana per la Lotta Contro i Tumori, Section of Milan, Italy \\ ${ }^{13}$ IFOM Fondazione Istituto FIRC di Oncologia Molecolare, Milano, Italy
}

Despite the excellent survival rate of Wilms tumor (WT) patients, only approximately one-half of children who suffer tumor recurrence reach second durable remission. This underlines the need for novel markers to optimize initial treatment. We investigated 77 tumors using Illumina 370CNV-QUAD genotyping BeadChip arrays and compared their genomic profiles to detect copy number $(\mathrm{CN})$ abnormalities and allelic ratio anomalies associated with the following clinicopathological variables: relapse (yes vs. no), age at diagnosis ( $\leq 24$ months vs. $>24$ months), and disease stage (low stage, I and II, vs. high stage, III and IV). We found that $\mathrm{CN}$ gains at chromosome region Iq2I.I-q3I.3 were significantly associated with relapse. Additional genetic events, including allelic imbalances at chromosome arms Ip, Iq, 3p, 3q, and I4q were also found to occur at higher frequency in relapsing tumors. Interestingly, allelic imbalances at IP and I4q also showed a borderline association with higher tumor stages. No genetic events were found to be associated with age at diagnosis. This is the first genome wide analysis with single nucleotide polymorphism (SNP) arrays specifically investigating the role of genetic anomalies in predicting WT relapse on cases prospectively enrolled in the same clinical trial. Our study, besides confirming the role of Iq gains, identified a number of additional candidate genetic markers, warranting further molecular investigations. @ $\quad 2012$ Wiley Periodicals, Inc.

\section{INTRODUCTION}

Thanks to an effective integration between surgery, chemotherapy, and radiotherapy, the survival rate of patients affected with Wilms tumor (W'T), the most frequent pediatric renal cancer, is now close to $90 \%$. This modern approach represents a model of multimodal therapy for cancer (Green et al., 1998; Tournade et al., 2001; de Kraker et al., 2004; Mitchell et al., 2006). However, only approximately half of children who suffer a tumor relapse reach second durable remission. This underlines the need of prognostic factors, either molecular and/or clinical, to guide clinicians to timely select patients at higher risk of relapse for more intense treatments.
Cornerstone prognostic factors in W'T remain tumor stage and the presence of diffuse anaplasia, both in pretreated and in chemotherapy-naive

\footnotetext{
Additional Supporting Information may be found in the online version of this article.

Supported by: Italian Association for Cancer Research (AIRC); Associazione Bianca Garavaglia, Busto Arsizio, Varese; POCEMON, Grant number: FP7-ICT-2007-216088; HYPERGENES, Grant number: HEALTH-F4-2007-201550.

*Correspondence to: Daniela Perotti, Department of Preventive and Predictive Medicine, Unit of Molecular bases of genetic risk and genetic testing, Fondazione IRCCS Istituto Nazionale dei Tumori, c/o AMADEOLAB, Via Amadeo 42, 20133 Milano, Italy. E-mail: daniela.perotti@istitutotumori.mi.it

Received 7 October 2011; Accepted 1 February 2012

DOI 10.1002/gcc.21951

Published online in

Wiley Online Library (wileyonlinelibrary.com).
} 
cases (Faria et al., 1996), and blastemal-type tumor after primary chemotherapy (Vujanic et al., 2002). Indeed, the principle of using disease stage and presence of diffuse anaplasia to select postnephrectomy chemotherapy intensity and radiotherapy remained basically unchanged during the two consecutive national WT trials carried out by the Italian Association of Pediatric Hematology and Oncology (AIEOP).

Different studies, aimed at characterizing the molecular genetics of WTs that are likely to relapse, led to diverse, although in some cases partially overlapping, results. Studies of loss of heterozygosity (LOH), performed using microsatellite markers, reported an association of $\mathrm{LOH}$ at chromosome arms 1p, 11q, 16q, and 22q with an adverse outcome (Grundy et al., 1994, 2005; Klamt et al., 1998; Messahel et al., 2009; Wittmann et al., 2007). Comparative genomic hybridization (CGH) to metaphase chromosomes displayed an increased frequency of $1 \mathrm{q}$ gains in recurring tumors (Hing et al., 2001), an observation consistent with the overexpression of $1 \mathrm{q}$ genes assessed by comparative expressed sequence hybridization (CESH) (Lu et al., 2002). Finally, microarray-based $\mathrm{CGH}(\mathrm{aCGH})$ at a genome wide resolution of approximately $1 \mathrm{Mb}$, performed on 76 WT samples enriched for recurred cases, identified gains on $1 \mathrm{q}$ as negative prognostic factors and other anomalies, including deletions on $12 \mathrm{q}$ and $18 \mathrm{q}$, as associated with relapse (Natrajan et al., 2006b). Different wholegenome studies have been performed on WTs using Affymetrix single nucleotide polymorphism (SNP) array platforms ranging from 10K (Yuan et al., 2005), to 250K, (Ohshima et al., 2009; Williams et al., 2010; Hawthorn and Cowell, 2011). However, none of these studies was specifically aimed at investigating the relation between the observed chromosomal abnormalities and patients' outcome.

We performed a genomic profiling by wholegenome SNP arrays, with an average resolution of $8 \mathrm{~Kb}$, on a group of prospectively enrolled WT patients, in order to identify and/or validate chromosomal regions showing copy number abnormalities (CNAs) and allelic ratio anomalies in tumor DNA, associated with clinical parameters. We took advantage of the renewed structure of the AIEOP WT Working Group, involving also an organized bio-banking project, which provided a unique opportunity to run biological studies and correlations with clinical data for patients consecutively registered into clinical trials.

\section{PATIENTS AND METHODS}

\section{Patients}

The study included 70 WT patients prospectively enrolled into the ongoing AIEOP WT-2003 protocol, and seven relapsing cases from the previous AIEOP W'T-1992 protocol. AIEOP-W'T2003 is a multicenter observational protocol, registering all patients under 18 years of age at the time of diagnosis of any primitive renal tumor. In WT, the main therapeutic strategy relays on up-front nephrectomy followed by adjuvant chemotherapy and radiotherapy, depending on tumor stage and presence of diffuse anaplasia as described elsewhere (Spreafico and Fossati-Bellani, 2006). Preoperative chemotherapy was administered only in selected cases at higher risk of intraoperative tumor rupture. The review boards of the institutions (IRBs) registering the patients approved the protocol. A specific informed consent to the use of biological samples for the aim of the study was obtained from the parents or legal tutors of all enrolled patients.

Of the 77 patients enrolled, 34 were males and 43 females, with a median age at diagnosis of 46 months (range 2-172 months). The distribution of the patients according to the age at diagnosis, tumor stage, and presence of diffuse anaplasia is reported in Supporting Information Table 1. Stage $\mathrm{V}$ patients were excluded, since bilateral WT are considered as tumors at high risk of relapse by definition. The median time to relapse in 17 relapsing patients was 10 months (range 1-37 months), one patient relapsed at 120 months; the median relapse-free time in the 59 nonrelapsing patients was 70 months (range 54-84 months).

\section{Survival Analysis}

The patterns of relapse free survival (RFS), defined as the time elapsed from surgery to the first evidence of tumor relapse/progression were estimated by means of the Kaplan-Meier method (Kaplan and Meier, 1958).

In order to assess the prognostic role of the available clinical variables, a univariate Cox regression model (Marubini and Valsecchi, 1995) was implemented for each of them (i.e., tumor stage, patient age at tumor onset, diffuse anaplasia). In this model each regression coefficient represents the logarithm of the hazard ratio (HR). The hypothesis of HR $=1.00$ was tested using the Wald Statistic. The variable age was dichotomized according to the cut-off of 24 months, 
and the tumor stage was categorized as low stage disease (Stages I and II) and high stage disease (Stages III and IV). This choice was due to the similar chemotherapy regimens received (vincristine/actinomycin D for Stages I and II and vincristine/actinomycin D/doxorubicin for Stages III and IV).

The role as prognostic factors of chromosomal abnormalities found preferentially associated with tumors of relapsing patients, irrespective of the above clinical parameters, was evaluated using a multivariate Cox regression model. All statistical analyses were performed with the SAS software (Version 9.2; SAS Institute Inc. Cary, NG).

\section{Genotyping}

DNA was extracted from tumor tissue fragments sampled by a pathologist following the procedures already reported (Perotti et al., 2004). DNA samples were analyzed using Illumina 370CNV-QUAD genotyping BeadChip arrays $(370 \mathrm{~K})$. The Infinium II Genotyping reaction steps were performed according to the manufacturer's specifications (Illumina, San Diego, CA) at the CBM genotyping service, Trieste, Italy (available at: http://www.cbm.fvg.it/services/genotyping?l=en). Normalized bead intensity data obtained for each sample were analyzed with Illumina Genome Studio v1.0.2 software that generates SNP genotypes from fluorescent intensities using the manufacturer's default cluster settings, together with the normalized measure of the total signal intensity for the two alleles of a SNP, defined as the $\log R$ ratio (LRR) and the normalized measure of the allelic intensity ratio of the two alleles, defined as the B allele frequencies (BAF), which were used to detect CNAs and allelic ratio anomalies.

\section{Generation of Copy Number and Allelic Event Calls, Quality Control and Comparisons}

The SNPRank Segmentation algorithm within Nexus Copy Number ${ }^{\text {TM }}$ v5 (Biodiscovery Inc., E1 Segundo, CA) was used to process LRR and BAF and perform the $\mathrm{CN}$ and allelic event calls (Parris et al., 2010; Veenma et al., 2010; Voortman et al., 2010; Halldorsdottir et al., 2011). Details of algorithm features and of set analytical parameters are provided as online Supporting Information.

We defined low-level CN gain and loss when LRR values varied from 0.25 to 0.6 or from -0.25 to -0.7 , respectively, high level $\mathrm{CN}$ gain when LRR values were $>0.6$ and homozygous copy loss when LRR values were $<-0.7$. Nexus automatically classifies three types of allelic ratio anomalies on the basis of BAF and of the homozygous frequency threshold (i.e., the minimum percentage of the probes that in a region must fall within a predefined BAF range): $\mathrm{LOH}$, allelic imbalance, and total allelic loss. We set a homozygous frequency threshold of $95 \%$ and called an $\mathrm{LOH}$ (i.e., the loss one allele) when BAF values were $>0.8$ or $<0.2$ and an allelic imbalance (i.e., maintenance of both alleles, but with an altered ratio) when BAF values varied from 0.2 to 0.4 or from 0.6 to 0.8 . Finally, we called a total allelic loss (i.e., loss of both alleles) if the probes showed a noncanonical profile with bands corresponding to the different possible genotypes.

A paired comparison analysis on $\mathrm{CN}$ and allelic ratios data was performed to identify aberrant regions associated with the phenotypes under investigation (relapse, age, stage) with the ad hoc comparison tool provided by Nexus v5. This tool allows comparing groups of profiles to look for regions with significantly different frequency between the groups (minimum difference threshold: $15 \%, P$ value threshold: 0.05 .) In this analysis LOH events are tagged as allelic losses. The software provides the Q-bound value that adjusts for multiple testing by performing False Discovery Rate (FDR) correction. Due to the exploratory nature of this study, a $Q$-bound value cutoff of 0.2 was set to define possible biologically relevant associations.

All the annotation and map information were based on the hg18 release of the human genome, and were retrieved from databases linked to Nexus (e.g. UCSC, Ensembl, miRNA database, Database of Genomic Variants, RepeatMasker).

\section{RESULTS}

\section{Survival Analysis}

Among the 77 patients included in the study, there were 18 relapses and 4 deaths, with a 6-year probability of RFS and overall survival of 0.78 and 0.95 , respectively (median followup time, 71 months; range, 36-211 months). Two relapses occurred in 24 patients younger than 24 months at diagnosis (8\%), and 16 in 53 patients older than 24 months at diagnosis $(30 \%)$; 9 relapses were in 55 Stage I and II patients (16\%), and 9 in 22 Stage III and IV patients (41\%).

Figure 1 shows the 6-year probability of RFS of the 77 patients according to age at diagnosis and 

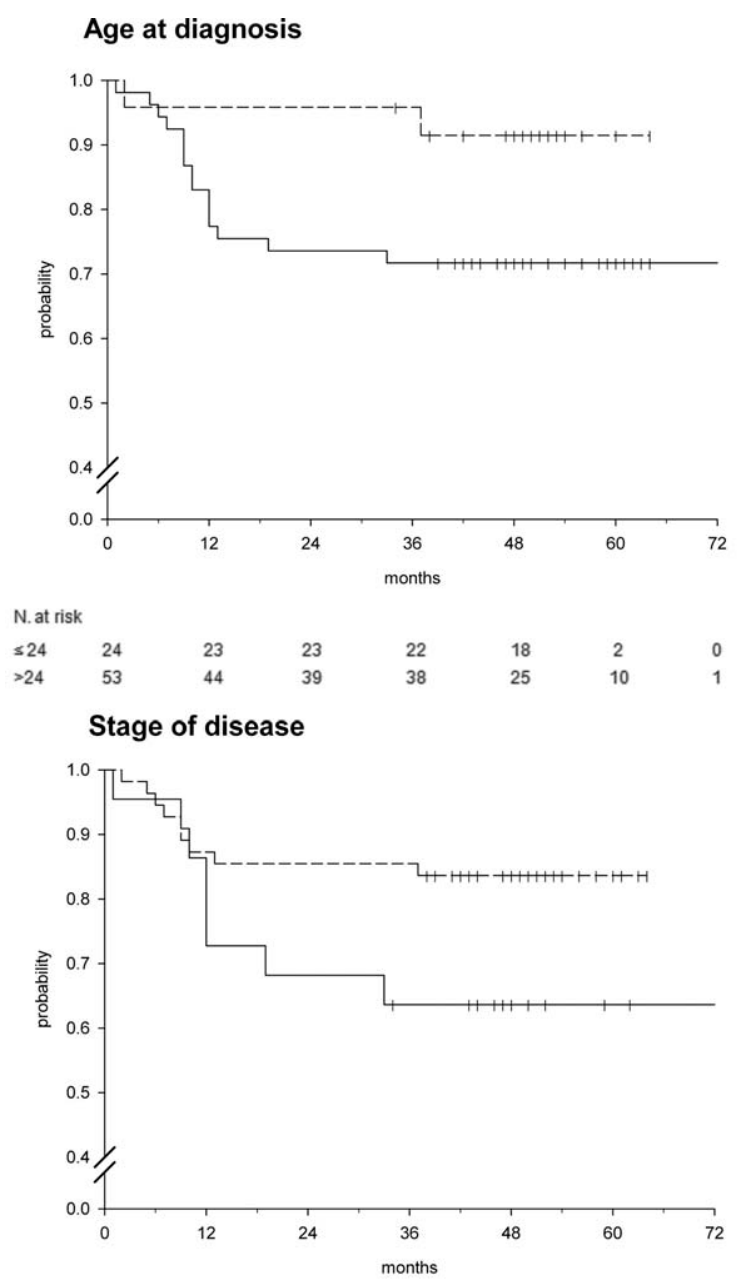

$\begin{array}{llllllll}\text { N. at risk } & & & & & & & \\ \text { I+II } & 55 & 48 & 47 & 47 & 35 & 10 & 0 \\ \text { III+IV } & 22 & 19 & 15 & 13 & 8 & 2 & 1\end{array}$

Figure I. Six years probability of relapse free survival (RFS) of the 77 patients according to age at diagnosis and disease stage. Upper panel: the dashed line represents the RFS of patients $\leq 24$ months of age at diagnosis and the continuous line the RFS of patients $>24$ months of age at diagnosis. Lower panel: the dashed line represents the RFS of patients with low stage (I and II) and the black line the RFS of patients with high stage (III and IV) disease. $N$, at risk, number of patients considered for each indicated time interval.

tumor stage. Table 1 reports the results of univariate Cox regression analysis for the considered clinical variables. Age at diagnosis $(P=0.078)$ and stage of disease $(P=0.081)$ had a borderline prognostic significance for RFS, whereas we did not observe a prognostic role of diffuse anaplasia.

\section{Chromosomal Regions Involved in CNAs and Allelic Ratio Anomalies}

The distribution of the chromosomal anomalies detected by the SNP array analysis in the examined samples is shown in Figure 2. Highly fre- quent genetic events, both CNAs and allelic ratio anomalies, visible as peaks in Figure 2 were observed in regions where constitutional CN variations (CNVs) are located, including chromosome segments $3 \mathrm{q} 26.1,4 \mathrm{q} 13.2$ and $4 \mathrm{q} 26,5 \mathrm{q} 13.2$, 6p21.32, and 6p21.33, 8p11.23-p11.22, 8q24.3, 9p11.2, 15q11.1-q11.2, 16p13.3, 18q12.3, 20q11.1, 21p11.2-p11.1 (the Database of Genomic Variants, available at: http://projects.tcag.ca/variation/). Excluding these regions, the chromosome arms most frequently involved in $\mathrm{CN}$ gains and losses were $1 \mathrm{q}(16 \%$ of cases) and $1 \mathrm{p}(14 \%), 7 \mathrm{p}(14 \%)$, $11 \mathrm{q}(9 \%), 14 \mathrm{q}(16 \%), 16 \mathrm{q}(13 \%), 17 \mathrm{p}(9 \%)$, and 22q $(9 \%)$, respectively. Most frequent allelic ratio anomalies were LOH on chromosome arms 1p $(12 \%), 11 \mathrm{p}(30 \%), 11 \mathrm{q}(13 \%), 16 \mathrm{q}(14 \%)$, and $22 \mathrm{q}$ (12\%), and imbalances on chromosome arms 1q (25\%), 6p (16\%), 6q (18\%), 12 (17\%), and 13q (17\%).

Among the focal aberrations detected, a few were already described in WT. In five samples we observed focal anomalies, ranging from 507 to $825 \mathrm{~kb}$ in length, involving the $M Y C N$ gene region on chromosome 2. Four W'Ts (one anaplastic and three with favorable histology) showed both low level CN gains and allelic imbalances involving partially overlapping regions (chr2:15,360,821-16,184,933; chr2:15,495, 567-16,097,299; chr2:15,495,567-16,243,938; chr2:15, 674,238-16,493,632; Supporting Information Fig. 1), while one tumor showed an allelic imbalance only (chr2:15,616,596-16,123,355). In two cases we observed homozygous deletions at 2q37.1, occurring within a wider region of $\mathrm{LOH}$, and detected as both homozygous copy loss, based on LLR values, and total allelic loss, based on BAF values (Supporting Information Fig. 2). In one sample, WT126, the homozygous deletion spanned approximately $1.4 \mathrm{Mb}$ (chr2:232,733,847-234,154,262) and involved 24 genes, including DIS3L2 and the microRNA gene miR-562. In the other case, WT227, the deletion was much smaller, encompassing approximately $50 \mathrm{~kb}$ (chr2:232,699,872-232,749,125), and involved the DIS3L2 and miR-562 genes only. To the best of our knowledge, this is the smallest homozygous deletion involving miR-562 reported to date in a WT (Drake et al., 2009). This finding provides further support to the hypothesis that the loss of this microRNA, which regulates EYA1, a critical gene for renal development, may contribute to W'T development.

\section{Chromosomal Abnormalities and Relapse}

The comparison of genomic profiles, for both CNAs and allelic ratio anomalies, in relapsing and 
TABLE I. Univariate Cox Models for Relapse Free Survival

\begin{tabular}{lccrr}
\hline & & \multicolumn{2}{c}{$95 \%$ Confidence interval } \\
\cline { 3 - 5 } Clinical variable & Hazard ratio & Lower limit & Upper limit & $P$ \\
\hline Age at diagnosis $(>24$ vs. $\leq 24$ months) & 3.776 & 0.863 & 16.520 & 0.078 \\
Stage (III + IV vs. I + II) & 2.334 & 0.900 & 6.058 & 0.08 I \\
Diffuse anaplasia (yes vs. no) & 0.654 & 0.087 & 4.936 & 0.68 I \\
\hline
\end{tabular}

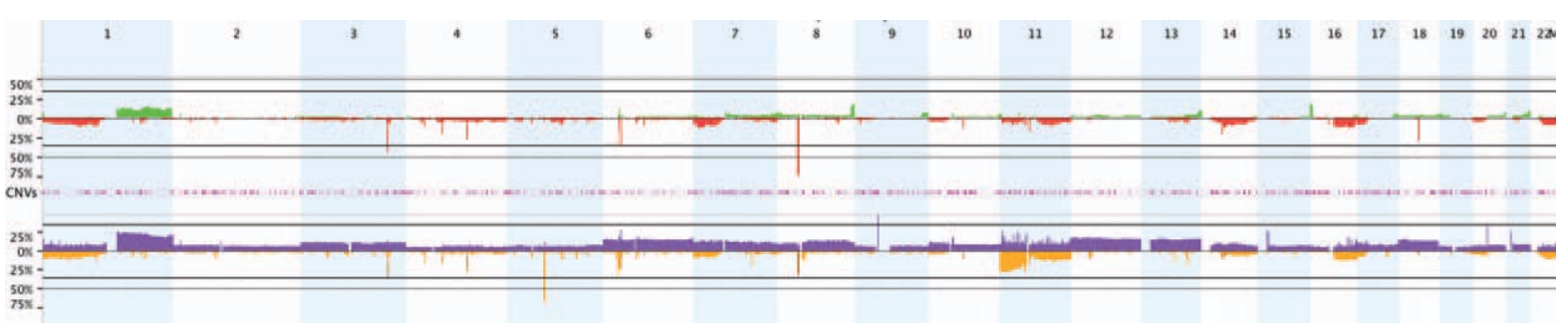

Figure 2. CNA frequency plot (upper panel) and allelic ratio anomaly frequency plot (lower panel) of the 77 WT patients. The percentage of samples with an aberration at a specific region along the genome is indicated along the $y$-axis. $\mathrm{CN}$ gains are plotted in green above the baseline, $\mathrm{CN}$ losses in red below the baseline. Allelic imbalances are plotted in purple above the baseline, allelic losses in brown below the baseline. The position of constitutional CNVs is indicated in pink.

nonrelapsing patients, is graphically shown in Figure 3. Regions with a significant difference in the frequency of aberrations between the two groups, using the specified parameter thresholds (minimum difference $>15 \%$; $P$ value $<0.05$ ), are reported in detail in Supporting Information Table 2. Tumors from relapsing patients showed a higher frequency of $\mathrm{CN}$ gains at different regions on chromosome segment 1q21.1-q44 and of CN losses on chromosome segments 1p36.32-p36.31, 1p36.21, 1p34.1, 1p32.3, 1p32.2-p31.3, 1p31.1-p22.3, 1p21.1, 16q22.1-q22.2 and 16q23.3-q24.2. A higher frequency of allelic losses was observed at regions on chromosome segments 1p36.33-p36.31, 1p36.21, 1p36.13, 1p36.12, 1p36.11, 1p35.2, 1p34.2, 1p33-p32.3, 1p32.2, 1p32.1, 1p31.3, 1p31.1-p22.3, 1p21.1, 5q13.2, 6p22.1, 11p14.3, 12q12, 16q22.1 and of allelic imbalances on chromosome segments 1p36.33, 1p36.32, 1p35.3, 1p31.1, 1p22.3, 1p22.2, 1p13.3, 1q21.1-q42.2, $1 \mathrm{q} 42.3, \quad 1 \mathrm{q} 43, \quad 1 \mathrm{q} 44,3 \mathrm{p} 26.3-\mathrm{p} 11.2,3 \mathrm{q} 11.2-\mathrm{q} 23$, 3q25.1-q25.2, 3q26.1, 6p21.32, 11p15.4, 11p12, 14q12-q21.1, 14q21.2, 14q22.1-q24.3, 16q13, 16q23.1, 16q24.2, and 21q11.2.

After adjustment for multiple testing by FDR correction, the only differences between the two groups of patients that showed $Q$-values below the defined threshold for biological relevance (range: 0.006-0.18) were those observed in the frequency of $\mathrm{CN}$ gains at 11 regions encompassing two distinct contigs mapped to 1q21.1-q31.3 (chr1:142,649,580$146,361,657$ and chr1:146,391,799-193,426,615). These regions were found to be gained in $28 \%(n=$
5) to $39 \%(n=7)$ of 18 relapsing patients vs. $0 \%$ to $5 \%(n=3)$ of 59 non relapsing patients. Interestingly, the two identified contigs overlap a 1q22-q25 interval of $\mathrm{CN}$ gains detected by aCGH that showed the highest level of association with relapse among regions amplified on $1 \mathrm{q}$ in W'Ts (Natrajan et al., 2006b).

A multivariate analysis performed including the $1 \mathrm{q}$ region with the highest level of association with relapsing patients (chr.1:148,129,525148,150,365; $Q$-bound $=0.006$ ), dichotomized as "gain" vs. "no gain," age at diagnosis, tumor stage, and presence of diffuse anaplasia, showed $1 \mathrm{q}$ gain as a prognostic factor independent from the clinical parameters $(P=0.006)$ (Supporting Information Table 3).

A few of the other identified anomalies preferentially associated with tumors from relapsing patients were focal and scattered along the genome and, therefore, might represent spurious observations, while others clustered at specific chromosomal arms encompassing large segments. The latter included allelic imbalances at different regions on chromosome arms 1p, 1q, 3p, 3q, and 14q (Q-bound range: $0.46-0.48)$.

\section{Chromosomal Abnormalities and Other Clinicopatholological Variables}

Genomic profiles, for both CNAs and allelic ratio anomalies, were then compared between the patients with low stage disease (I and II) and 


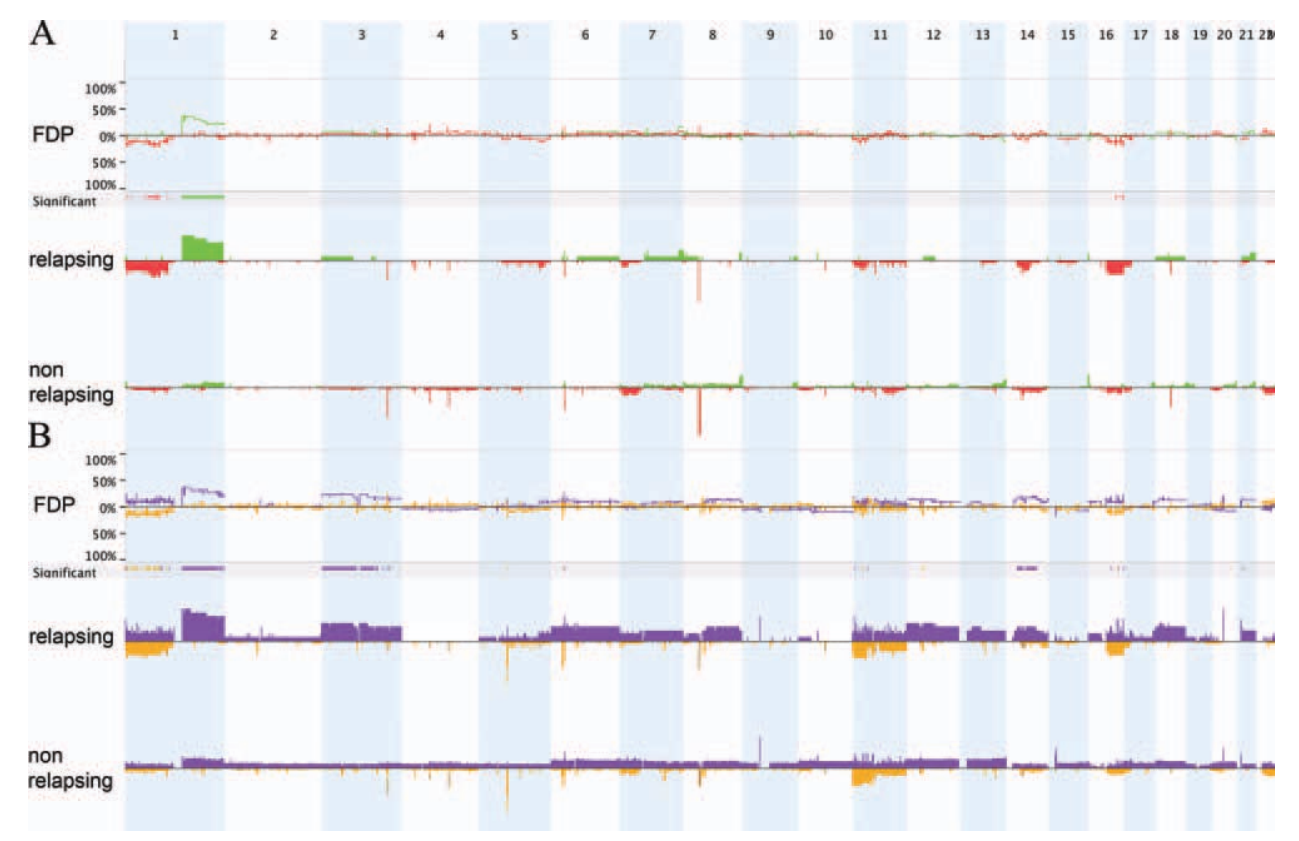

Figure 3. Identification of chromosomal regions with significant difference in the frequency of aberrations between relapsing and nonrelapsing patients. Frequency difference plots (FDPs) between the two groups considered, as well as the frequency plots of each group, are shown for both CNAs (A) and allelic ratio anomalies (B). CN gains and losses and allelic imbalances and losses are plotted in colors as in Figure 2. In the CNA FDP, if the magnitude of a gain or a loss is greater in the relapsing tumor group, then the gain is plotted in green above the baseline and the loss is plotted in red below the baseline and vice versa. In the allelic event

those with high stage disease (III and IV). Regions with a significant difference in the frequency of genetic alterations between the two groups after the initial statistical analysis are reported in detail in Supporting Information Table 4 and graphically shown in Supporting Information Figure 3. CN gains at regions on chromosome segments 1q21.1, 1q21.2, 1q42.13q43, 1q44, 7q11.22, CN losses on chromosome segments 1p31.1, 1p22.3, 11q22.1, allelic losses on chromosome segments 1p36.32, 1p31.1, 2p12, $5 q 13.2,7 q 22.1,7 q 31.1$, and allelic imbalances on chromosome segments 1p36.33, 1p36.32-p36.11, 1p35.3, 1p34.2, 1p34.1, 1p33, 1p32.3, 1p32.2, 1p31.3, 1p31.1, 1p22.3-p22.1, 1p21.3, 1p21.2, 1p21.1，1p13.3，1p13.2-p11.1，2p24.3，6p21.33， $14 q 11.2,14 q 12-q 21.1,14 q 22.1-q 32.32,17 \mathrm{p} 13.2$, $17 \mathrm{p} 13.1,17 \mathrm{p} 11.2,22 \mathrm{q} 13.32$ were more frequently observed in high stage disease patients, while allelic losses at $6 \mathrm{p} 22.1$ were found with higher frequency in low stage disease cases.

After FDR correction, a borderline level of relevance $(Q$-bound $=0.21)$ was observed for allelic imbalances at several regions on chromosome arms 1p (1p36.33, 1p36.32, 1p36.31-p36.23, 1p36.22, 1p36.21, 1p36.13, 1p36.12, 1p35.3, 1p33,
FDP, if the magnitude of an allelic imbalance or allelic loss is greater in relapsing tumor group, then the allelic imbalance is plotted in purple above the baseline and the allelic loss in brown below the baseline and vice versa. Marks on the "significant" tracks displayed below the FDPs indicate regions of significant difference between the two groups based on the set parameters (minimum difference threshold: I5\%, $P$ value threshold: 0.05 ). In (A) red bars depict regions of $\mathrm{CN}$ loss and green bars regions of $\mathrm{CN}$ gain. In (B) purple bars depict regions of allelic imbalance and brown bars regions of allelic loss.

1p31.1, 1p22.3, 1p22.2, 1p22.1, 1p13.3, 1p13.2p11.1) and $14 q(14 q 13.2,14 q 22.1,14 q 24.1-q 32.32)$, which were found to be involved in anomalies in 18 to $36 \%$ of patients with high stage disease vs. 0 to $7 \%$ of patients with low stage disease.

Regions showing a significant difference in the frequency of genetic alterations between patients with an age at diagnosis $\leq 24$ months and those with an age at diagnosis $>24$ months after the initial statistical analysis are detailed in Supporting Information Table 5 and graphically shown in Supporting Information Figure 4. CN gain at chromosome segment 1q31.3-q32.1, allelic imbalances at chromosome segments 1q21.1, 1q21.3-q24.1, 1q25.1, 1q31.1, 1q31.2-q31.3, 1q32.1-q42.13, $1 q 42.2$, and $1 q 43$ were more frequent in older patients, while CN loss at 4q13.2 and allelic losses at $11 \mathrm{p} 15.1,11 \mathrm{p} 11.2$, and $17 \mathrm{q} 23.2$ were detected with higher frequency in younger patients. However, none of these associations maintained statistical significance after FDR correction.

\section{DISCUSSION}

A fundamental goal of modern pediatric oncology trials in WT is how to better tailor the 
intensity of therapy to the risk of treatment failure. Previous studies in WT identified different regions of chromosomal gain/loss proposed as prognostic/predictive factors (Grundy et al., 1994, 2005; Klamt et al., 1998; Messahel et al., 2009; Natrajan et al., 2006b; Wittmann et al., 2007). Among them, the only factor currently used in clinical practice to stratify WT patients and to treat them accordingly, is a combined LOH at $1 \mathrm{p}$ and 16q (Perlman, 2005). These biomarkers allow identifying a subset of favorable-histology WT patients who have a significantly increased risk of relapse and death, as supported by the National Wilms Tumor Study-5 NWTS-5 trial (Grundy et al., 2005). However, according to that study, the proportion of patients predicted to relapse solely on the basis of the concomitant occurrence of $\mathrm{LOH}$ at both loci was relatively low comparing with the whole number of actually relapsing patients. More recently, a study from the United Kingdom Children's Cancer and Leukemia Group confirmed the prognostic impact of $16 \mathrm{q}$ $\mathrm{LOH}$ in 426 favorable-histology WTs, while 1p LOH showed no significant association with RFS (Messahel et al., 2009). LOH analyses performed using microsatellite markers identified further regions proposed to be associated with a poor outcome, including 11q and 22q (Klamt et al., 1998; Wittmann et al., 2007). While studies focusing on $1 \mathrm{p}$ and $16 \mathrm{q} \mathrm{LOH}$ accrued a large cohort of patients (Grundy et al., 2005; Messahel et al., 2009), those on 11q and 22q LOH need further validation due to the relatively small number of investigated cases (Klamt et al., 1998; Wittmann et al., 2007).

Here, we performed a genome wide scan for CNAs and allelic ratio anomalies with the specific aim to identify chromosomal regions associated with clinical variables including relapse, disease stage, and age at diagnosis. This study adds some new aspects to the scenario of the already published genome wide high-resolution analyses performed to search for chromosomal regions associated with relapse in WT (Hing et al., 2001; Natrajan et al., 2006b, 2007).

In fact, our study represents the first whole genome analysis performed on cases for the majority prospectively enrolled in the same controlled clinical trial, while previously reported studies were carried out on series of patients treated according to different protocols and enriched for relapsing cases (Hing et al., 2001; Natrajan et al., 2006b, 2007). In addition, this is the first high-resolution analysis investigating the role of CNAs in predicting WT relapse performed with a commercially available platform, while previously published studies have been carried out with CGH on metaphases (Hing et al., 2001) or on home-made BAC clone arrays (Natrajan et al., 2006b, 2007). This will allow independent validation studies to be performed using the same platform and the same standardized conditions. Furthermore, the choice of a high resolution whole-genome SNP array platform allowed us to also detect, in addition to $\mathrm{CN}$ gains and $\mathrm{CN}$ losses, genetic events affecting allelic ratio, including allelic losses and imbalances, which do not necessarily lead to CNAs. The use of a dedicated segmentation algorithm (Nexus Copy Number ${ }^{\mathrm{TM}}$ ) on raw fluorescence data allowed us to define at a high resolution the boundaries of the regions most significantly associated with the clinical variables investigated. Such an algorithm is widely used for CNAs and allelic ratio anomalies detection, particularly in the field of cancer genome profiling (Chen et al., 2011; Duy et al., 2011; Halldorsdottir et al., 2011; Ismail et al., 2011; Paulson et al., 2011) with an overall high rate of consensus calls across studies that supports its accuracy (Darvishi, 2010; Matsuzaki et al., 2009). Although it has been recently reported that Nexus may be affected with an overcall of CNAs, especially with more relaxed analysis parameters (Dellinger et al., 2010) in the present study, the use of Nexus with conservative parameters (in particular, as for the significance threshold and the minimum number of probes per segment), allowed us to find a good trade off between sensitivity and specificity in CNAs detection (Darvishi, 2010).

We found that CN gains at different regions on chromosome segment 1q21.1-q31.3 were significantly associated with relapse in WT patients, consistent with previously published observations (Hing et al., 2001; Natrajan et al., 2006b). Therefore, our results represent a validation of such data using a different analytical approach. The 1q21.1-q31.3 region identified by our approach contains a number of genes whose expression has been already characterized in WTs in association with relapse. The expression levels of $R A B 25$, $H D G F, C R A B P 2, N E S$, and NTRK1, promising candidate cancer genes on the basis of previous studies on WTs and other tumor types (Cheng et al., 2004; Donovan et al., 1994; Williams et al., 2004; Li et al., 2005), were found not to be correlated with CNAs and relapse (Natrajan et al., 2006b). On the other hand, the overexpression 
and amplification of CACNA1E, a gene encoding the ion-conducting $\alpha 1$ subunit of voltage dependent R-type calcium channels, has been correlated with relapse in WTs (Natrajan et al., 2006a). Other 1q genes that have been found to be overexpressed in relapsing WTs are PMF1, SETDB1, SLC39A1, SMG5, SEMA6C, ENSA, ADAM15, DPM3, RAG1AP1, CD1D, WDR424A, PBX1, UfC1, QSCN6, VAMP4, KIAAO492 (Huang et al., 2009). Among these, ADAM15 and SETDB1 seem the most interesting for a possible role in WT, since the overexpression of the metalloproteinase ADAM15 is associated with the progression of prostate and breast cancer (Kuefer et al., 2006), and SETDB1, an enzyme that methylates histone $\mathrm{H} 3$ on lysine 9, has been found to be amplified in melanoma, and overexpressed in nonsmall cell lung cancer cell lines (Ceol et al., 2011; Watanabe et al., 2008).

Interestingly the 1q region enriched for CNAs preferentially associated with relapse contains regions reported as constitutionally polymorphic in copy number (the Database of Genomic Variants, available at: http://projects.tcag.ca/variation/). This suggests that also germline CNVs might have an impact on relapse in WT. To verify this possibility, further analyses on the normal DNA of WT patients are needed.

The positive and negative predictive values associated with $\mathrm{CN}$ gains at the above chromosome 1q regions ranged between 0.667 and 1.00 (median, 0.778 ) and between 0.819 and 0.843 (median, 0.836 ), respectively. However, the pertinent sensitivity was relatively low (38.8\%; range, $27.7 \%-$ $38.8 \%$ ), implying that other molecular alterations are likely to be associated with tumor relapse. Consistently, a few genetic events clustering at specific chromosomal arms were found to occur at higher frequency in relapsing tumors, including allelic imbalances at $1 \mathrm{p}, 1 \mathrm{q}, 3 \mathrm{p}, 3 \mathrm{q}$ and $14 \mathrm{q}$, although with $Q$-values $>0.2$. The preferential association of allelic imbalances at $1 \mathrm{q}$ parallels that observed for 1q gains, indicating that the latter preferentially involve a single allele. The higher frequency of allelic imbalances at $1 \mathrm{p}$ in tumors of relapsing patients is consistent with previous investigations that showed an association with worse prognosis of allelic alterations at this chromosome arm, detected as $\mathrm{LOH}$ by conventional microsatellite analyses (Grundy et al., 1994, 2005).

Remarkably, the association with allelic imbalances involving most of chromosomes 3 and $14 \mathrm{q}$ represents a novel report, which warrants further investigations.
We also observed a preferential association between relapse and anomalies on 16q. However, as they involved a few very restricted and scattered regions we were unable to confirm formerly reported association with prognosis in WT patients, although this might be due to the relatively small sample set examined in our study compared with previous surveys (Grundy et al., 1994, 2005; Klamt et al., 1998; Messahel et al., 2009; Wittmann et al., 2007).

We found that allelic imbalances in a number of regions on chromosome arms $1 \mathrm{p}$ and $14 \mathrm{q}$ showed a borderline association with tumor Stages III and IV. This evidence suggests that these regions might contain genes involved in tumor progression, and would explain the higher frequency of these anomalies that we observed in relapsing patients, given the well-established association of high stage disease with poorer outcome. A few studies had previously suggested the importance of the age at diagnosis in the prognosis of WT together with other established factors. Children younger than 24 months at initial diagnosis and small-volume Stage I tumors were found to have a better outcome than older children (Green et al., 1993, 2001; Pritchard-Jones et al., 2003). Furthermore, lower frequencies of the putative adverse prognostic markers $1 \mathrm{p}$ and 16q LOH have been reported in children younger than 24 months with Stage I tumors weighing less than 550 grams (Grundy et al., 2005). We also observed that age at diagnosis ( $>24$ vs. $\leq 24$ months) had a prognostic role (HR $=3.776)$ although not statistically significant $(P=$ 0.078). This might suggest that WTs arising at different ages are pathologically distinct entities, with different aggressiveness. However, our analyses failed to provide molecular evidences supporting such hypothesis, since no genetic alteration preferentially associated with different ages of disease onset was observed.

Interestingly, the lack of preferential association of 1q CN gains with both tumor stage and age at diagnosis suggests that these anomalies might be markers of relapse independent from these clinical variables. This was confirmed by the results of the multivariate analysis.

In conclusion, our observations indicate that genome wide studies, performed on prospectively enrolled series of WT cases are able to identify molecular markers potentially useful to optimize clinical treatment. Our study provides useful new information for the selection of chromosomal regions potentially associated with prognosis in 
W'T, to be validated in prospectively enrolled cohorts of patients.

\section{ACKNOWLEDGMENTS}

The authors thank Dr. Soheil Shams (Biodiscovery Inc., El Segundo, CA) for assistance and troubleshooting in CNA analysis with Nexus v5 and Dr. James H. Fallon (Department of Psychiatry and Human Behavior, University of California, Irvine, CA) for the revision and proofreading of the manuscript.

\section{REFERENCES}

Ceol CJ, Houvras Y, Jane-Valbuena J, Bilodeau S, Orlando DA, Battisti V, Fritsch L, Lin WM, Hollmann TJ, Ferre F, Bourque C, Burke CJ, Turner L, Uong A, Johnson LA, Beroukhim R, Mermel CH, Loda M, it-Si-Ali S, Garraway LA, Young RA, Zon LI. 2011. The histone methyltransferase SETDB1 is recurrently amplified in melanoma and accelerates its onset. Nature 471:513-517.

Chen Z, Liu Z, Li W, Qu K, Deng X, Varma MG, Fichera A, Pigazzi A, Garcia-Aguilar J. 2011. Chromosomal copy number alterations are associated with tumor response to chemoradiation in locally advanced rectal cancer. Genes Chromosomes Cancer 50:689-699.

Cheng KW, Lahad JP, Kuo WL, Lapuk A, Yamada K, Auersperg N, Liu J, Smith-McCune K, Lu KH, Fishman D, Gray JW, Mills GB. 2004. The RAB25 small GTPase determines aggressiveness of ovarian and breast cancers. Nat Med 10:1251-1256.

Darvishi K. 2010. Application of Nexus copy number software for CNV detection and analysis. Curr Protoc Hum Genet 4: 4.14.1-4.14.28.

de Kraker J, Graf N, van TH, Pein F, Sandstedt B, Godzinski J, Tournade MF. 2004. Reduction of postoperative chemotherapy in children with stage I intermediate-risk and anaplastic Wilms' tumour (SIOP 93-01 trial): A randomised controlled trial. Lancet 364:1229-1235.

Dellinger AE, Saw SM, Goh LK, Seielstad M, Young TL, Li YJ. 2010. Comparative analyses of seven algorithms for copy number variant identification from single nucleotide polymorphism arrays. Nucleic Acids Res 38:e105.

Donovan MJ, Hempstead B, Huber LJ, Kaplan D, Tsoulfas P, Chao M, Parada L, Schofield D. 1994. Identification of the neurotrophin receptors $\mathrm{p} 75$ and trk in a series of Wilms' tumors. Am J Pathol 145:792-801.

Drake KM, Ruteshouser EC, Natrajan R, Harbor P, Wegert J, Gessler M, Pritchard-Jones K, Grundy P, Dome J, Huff V, Jones C, Aldred MA. 2009. Loss of heterozygosity at 2q37 in sporadic Wilms' tumor: putative role for miR-562. Clin Cancer Res 15:5985-5992.

Duy C, Hurtz C, Shojaee S, Cerchietti L, Geng H, Swaminathan S, Klemm L, Kweon SM, Nahar R, Braig M, Park E, Kim YM, Hofmann WK, Herzog S, Jumaa H, Koeffler HP, Yu JJ, Heisterkamp N, Graeber TG, Wu H, Ye BH, Melnick A, Muschen M. 2011. BCL6 enables $\mathrm{Ph}+$ acute lymphoblastic leukaemia cells to survive BCR-ABL1 kinase inhibition. Nature 473:384-388.

Faria P, Beckwith JB, Mishra K, Zuppan C, Weeks DA, Breslow N, Green DM. 1996. Focal versus diffuse anaplasia in Wilms tumor-new definitions with prognostic significance: A report from the National Wilms Tumor Study Group. Am J Surg Pathol 20:909-920.

Green DM, Breslow NE, D'Angio GJ. 1993. The treatment of children with unilateral Wilms' tumor. J Clin Oncol 11:1009-1010.

Green DM, Breslow NE, Beckwith JB, Finklestein JZ, Grundy PE, Thomas PR, Kim T, Shochat SJ, Haase GM, Ritchey ML, Kelalis PP, D'Angio GJ. 1998. Comparison between single-dose and divided-dose administration of dactinomycin and doxorubicin for patients with Wilms' tumor: a report from the National Wilms' Tumor Study Group. J Clin Oncol 16:237-245.

Green DM, Breslow NE, Beckwith JB, Ritchey ML, Shamberger RC, Haase GM, D'Angio GJ, Perlman E, Donaldson M, Grundy PE, Weetman R, Coppes MJ, Malogolowkin M,
Shearer P, Coccia P, Kletzel M, Thomas PR, Macklis R, Tomlinson G, Huff V, Newbury R, Weeks D. 2001. Treatment with nephrectomy only for small, stage I/favorable histology Wilms' tumor: A report from the National Wilms' Tumor Study Group. J Clin Oncol 19:3719-3724.

Grundy PE, Telzerow PE, Breslow N, Moksness J, Huff V, Paterson MC. 1994. Loss of heterozygosity for chromosomes $16 \mathrm{q}$ and $1 \mathrm{p}$ in Wilms' tumors predicts an adverse outcome. Cancer Res 54:2331-2333.

Grundy PE, Breslow NE, Li S, Perlman E, Beckwith JB, Ritchey ML, Shamberger RC, Haase GM, D'Angio GJ, Donaldson M, Coppes MJ, Malogolowkin M, Shearer P, Thomas PR, Macklis R, Tomlinson G, Huff V, Green DM. 2005. Loss of heterozygosity for chromosomes $1 \mathrm{p}$ and $16 \mathrm{q}$ is an adverse prognostic factor in favorable-histology Wilms tumor: A report from the National Wilms Tumor Study Group. J Clin Oncol 23: $7312-7321$.

Halldorsdottir AM, Sander B, Goransson H, Isaksson A, Kimby E, Mansouri M, Rosenquist R, Ehrencrona H. 2011. High-resolution genomic screening in mantle cell lymphoma--specific changes correlate with genomic complexity, the proliferation signature and survival. Genes Chromosomes Cancer 50:113-121.

Hawthorn L, Cowell JK. 2011. Analysis of wilms tumors using SNP mapping array-based comparative genomic hybridization. PLoS One 6:e18941.

Hing S, Lu YJ, Summersgill B, King-Underwood L, Nicholson J, Grundy P, Grundy R, Gessler M, Shipley J, Pritchard-Jones K. 2001. Gain of $1 \mathrm{q}$ is associated with adverse outcome in favorable histology Wilms' tumors. Am J Pathol 158:393-398.

Huang CC, Gadd S, Breslow N, Cutcliffe C, Sredni ST, Helenowski IB, Dome JS, Grundy PE, Green DM, Fritsch MK, Perlman EJ. 2009. Predicting relapse in favorable histology Wilms tumor using gene expression analysis: A report from the Renal Tumor Committee of the Children's Oncology Group. Clin Cancer Res 15:1770-1778.

Ismail A, Bandla S, Reveiller M, Toia L, Zhou Z, Gooding WE, Kalatskaya I, Stein L, D'Souza M, Litle VR, Peters JH, Pennathur A, Luketich JD, Godfrey TE. 2011. Early G1 cyclin-dependent kinases as prognostic markers and potential therapeutic targets in esophageal adenocarcinoma. Clin Cancer Res 17:4513-4522

Kaplan EL, Meier P. 1958. Non-parametric estimation from incomplete observations. J Am Stat Assoc 3:457-481.

Klamt B, Schulze M, Thate C, Mares J, Goetz P, Kodet R, Scheurlen W, Weirich A, Graf N, Gessler M. 1998. Allele loss in Wilms tumors of chromosome arms 11q, I6q, and 22q correlates with clinicopathological parameters. Genes Chrom Cancer 22:287-294

Kuefer R, Day KC, Kleer CG, Sabel MS, Hofer MD, Varambally S, Zorn CS, Chinnaiyan AM, Rubin MA, Day ML. 2006. ADAM15 disintegrin is associated with aggressive prostate and breast cancer disease. Neoplasia 8:319-329.

Li W, Kessler P, Williams BR. 2005. Transcript profiling of Wilms tumors reveals connections to kidney morphogenesis and expression patterns associated with anaplasia. Oncogene 24:457-468.

Lu YJ, Hing S, Williams R, Pinkerton R, Shipley J, PritchardJones K. 2002. Chromosome 1q expression profiling and relapse in Wilms' tumour. Lancet 360:385-386.

Marubini E, Valsecchi MG. 1995. Analysing Survival Data from Clinical Trials and observational Studies. Chichester, UK: John Wiley \& Sons.

Matsuzaki H, Wang PH, Hu J, Rava R, Fu GK. 2009. High resolution discovery and confirmation of copy number variants in 90 Yoruba Nigerians. Genome Biol 10:R125.

Messahel B, Williams R, Ridolfi A, A'hern R, Warren W, Tinworth $\mathrm{L}$, Hobson $\mathrm{R}$, Al-Saadi $\mathrm{R}$, Whyman $\mathrm{G}$, Brundler MA, Kelsey A, Sebire N, Jones C, Vujanic G, Pritchard-Jones K. 2009. Allele loss at $16 \mathrm{q}$ defines poorer prognosis Wilms tumour irrespective of treatment approach in the UKW1-3 clinical trials: a Children's Cancer and Leukaemia Group (CCLG) Study. Eur J Cancer 45:819-826.

Mitchell C, Pritchard-Jones K, Shannon R, Hutton C, Stevens S, Machin D, Imeson J, Kelsey A, Vujanic GM, Gornall P, Walker J, Taylor R, Sartori P, Hale J, Levitt G, Messahel B, Middleton H, Grundy R, Pritchard J. 2006. Immediate nephrectomy versus preoperative chemotherapy in the management of non-metastatic Wilms' tumour: Results of a randomised trial (UKW3) by the UK Children's Cancer Study Group. Eur J Cancer 42: 2554-2562. 
Natrajan R, Little SE, Reis-Filho JS, Hing L, Messahel B, Grundy PE, Dome JS, Schneider T, Vujanic GM, PritchardJones K, Jones C. 2006a. Amplification and overexpression of CACNA1E correlates with relapse in favorable histology Wilms' tumors. Clin Cancer Res 12:7284-7293

Natrajan R, Williams RD, Hing SN, Mackay A, Reis-Filho JS, Fenwick K, Iravani M, Valgeirsson H, Grigoriadis A, Langford CF, Dovey O, Gregory SG, Weber BL, Ashworth A, Grundy PE, Pritchard-Jones K, Jones C. 2006b. Array CGH profiling of favourable histology Wilms tumours reveals novel gains and losses associated with relapse. J Pathol 210:49-58.

Natrajan R, Little SE, Sodha N, Reis-Filho JS, Mackay A, Fenwick K, Ashworth A, Perlman EJ, Dome JS, Grundy PE, Pritchard-Jones K, Jones C. 2007. Analysis by array CGH of genomic changes associated with the progression or relapse of Wilms' tumour. J Pathol 211:52-59.

Ohshima J, Haruta M, Arai Y, Kasai F, Fujiwara Y, Ariga T, Okita H, Fukuzawa M, Hata J, Horie H, Kaneko Y. 2009. Two candidate tumor suppressor genes, MEOX2 and SOSTDC1, identified in a 7 p21 homozygous deletion region in a Wilms tumor. Genes Chromosomes Cancer 48:1037-1050.

Parris TZ, Danielsson A, Nemes S, Kovacs A, Delle U, Fallenius G, Mollerstrom E, Karlsson P, Helou K. 2010. Clinical implications of gene dosage and gene expression patterns in diploid breast carcinoma. Clin Cancer Res 16:3860-3874.

Paulson V, Chandler G, Rakheja D, Galindo RL, Wilson K, Amatruda JF, Cameron S. 2011. High-resolution array CGH identifies common mechanisms that drive embryonal rhabdomyosarcoma pathogenesis. Genes Chromosomes Cancer 50:397-408.

Perlman EJ. 2005. Pediatric renal tumors: Practical updates for the pathologist. Pediatr Dev Pathol 8:320-338

Perotti D, De Vecchi G, Testi MA, Lualdi E, Modena P, Mondini P, Ravagnani F, Collini P, Di Renzo F, Spreafico F, Terenziani M, Sozzi G, Fossati-Bellani F, Radice P. 2004. Germline mutations of the POU6F2 gene in Wilms tumors with loss of heterozygosity on chromosome 7p14. Hum Mutat 24:400-407.

Pritchard-Jones K, Kelsey A, Vujanic G, Imeson J, Hutton C, Mitchell C. 2003. Older age is an adverse prognostic factor in stage I, favorable histology Wilms' tumor treated with vincristine monochemotherapy: A study by the United Kingdom Children's Cancer Study Group, Wilm's Tumor Working Group. J Clin Oncol 21:3269-3275.

Spreafico F, Fossati-Bellani F. 2006. Wilms' tumor: Past, present and (possibly) future. Expert Rev Anticancer Ther 6:249-258.
Tournade MF, Com-Nougue C, de KJ, Ludwig R, Rey A, Burgers JM, Sandstedt B, Godzinski J, Carli M, Potter R, Zucker JM. 2001. Optimal duration of preoperative therapy in unilateral and nonmetastatic Wilms' tumor in children older than 6 months: Results of the Ninth International Society of Pediatric Oncology Wilms' Tumor Trial and Study. J Clin Oncol 19:488500 .

Veenma D, Beurskens N, Douben H, Eussen B, Noomen P, Govaerts L, Grijseels E, Lequin M, de KR, Tibboel D, de KA, Van OD. 2010. Comparable low-level mosaicism in affected and non affected tissue of a complex CDH patient. PLoS One 5:e15348.

Voortman J, Lee JH, Killian JK, Suuriniemi M, Wang Y, Lucchi M, Smith WI, Jr., Meltzer P, Wang Y, Giaccone G. 2010. Array comparative genomic hybridization-based characterization of genetic alterations in pulmonary neuroendocrine tumors. Proc Natl Acad Sci USA 107:13040-13045.

Vujanic GM, Sandstedt B, Harms D, Kelsey A, Leuschner I, de Kraker J. 2002. Revised International Society of Paediatric Oncology (SIOP) working classification of renal tumors of childhood. Med Pediatr Oncol 38:79-82.

Watanabe H, Soejima K, Yasuda H, Kawada I, Nakachi I, Yoda S, Naoki K, Ishizaka A. 2008. Deregulation of histone lysine methyltransferases contributes to oncogenic transformation of human bronchoepithelial cells. Cancer Cell Int 8:15.

Williams RD, Al-Saadi R, Chagtai T, Popov S, Messahel B, Sebire N, Gessler M, Wegert J, Graf N, Leuschner I, Hubank M, Jones C, Vujanic G, Pritchard-Jones K. 2010. Subtype-specific FBXW7 mutation and MYCN copy number gain in Wilms' tumor. Clin Cancer Res 16:2036-2045.

Williams RD, Hing SN, Greer BT, Whiteford CC, Wei JS, Natrajan R, Kelsey A, Rogers S, Campbell C, Pritchard-Jones K, Khan J. 2004. Prognostic classification of relapsing favorable histology Wilms tumor using cDNA microarray expression profiling and support vector machines. Genes Chromosomes Cancer 41:65-79.

Wittmann S, Zirn B, Alkassar M, Ambros P, Graf N, Gessler M. 2007. Loss of $11 \mathrm{q}$ and $16 \mathrm{q}$ in Wilms tumors is associated with anaplasia, tumor recurrence, and poor prognosis. Genes Chromosomes Cancer 46:163-170.

Yuan E, Li CM, Yamashiro DJ, Kandel J, Thaker H, Murty VV, Tycko B. 2005. Genomic profiling maps loss of heterozygosity and defines the timing and stage dependence of epigenetic and genetic events in Wilms' tumors. Mol Cancer Res 3:493-502. 